\title{
Consequences of Neural Cell Adhesion Molecule Deficiency on Cell Migration in the Rostral Migratory Stream of the Mouse
}

\author{
Geneviève Chazal, ${ }^{1}$ Pascale Durbec, ${ }^{1}$ Aleksandar Jankovski, ${ }^{2}$ Geneviève Rougon, ${ }^{1}$ and Harold Cremer $^{1}$ \\ 1 Laboratoire de Génétique et Physiologie du Développement, Institut de Biologie du Développement de Marseille, Centre \\ National de la Recherche Scientifique/Institut National de la Santé et de la Recherche Médicale (INSERM)/Université de la \\ Méditerranée, Campus de Luminy, 13288 Marseille, France, and INSERM, Hôpital de la Salpétrière, 75651 Paris Cedex \\ 13, France
}

In vertebrates, interneurons of the olfactory bulb $(\mathrm{OB})$ are generated postnatally and throughout life at the subventricular zone of the forebrain. The neuronal precursors migrate tangentially through the forebrain using a well defined pathway, the rostral migratory stream (RMS), and a particular mode of migration in a chain-like organization. A severe size reduction of the OB represents the most striking morphological phenotype in neural cell adhesion molecule (NCAM)-deficient mice. This defect has been traced back to a migration deficit of the precursors in the RMS and linked to the lack of the polysialylated form of NCAM. In this study we investigate the morphological alterations and functional properties of the RMS in mice totally devoid of all isoforms of NCAM and polysialic acid (PSA). We show that a morphologically altered, but defined and continuous pathway exists in mutants, and we present in vivo and in vitro evidence that PSA-NCAM in the RMS is not essential for the formation and migration of chains. Instead, we find a massive gliosis associated with the formation of membrane specializations in a heterotypic manner, linking precursors to astrocytes. This finding and the over-representation and defasciculation of axons in the pathway suggest that important interactions between migrating cells and their stationary environment are perturbed in the mutants. Finally, we used transplantation experiments to demonstrate that lack of PSA-NCAM leads to a decrease but not a total blockade of migration and demonstrate that the mutant RMS is functional in transporting normal neuronal precursors to the OB.

Key words: cell adhesion molecules; subventricular zone; olfactory bulb; neurogenesis; tangential cell migration; polysialic acid
Most neurons in the brain are generated during embryonic and early postnatal stages. After leaving the cell cycle they migrate radial from their place of birth at ventricular zones to their final destinations using well described glia-guided mechanisms (Rakic, 1990). However, interneurons of the olfactory bulb are generated postnatally and throughout the entire lifetime of rodents at the subventricular zone (SVZ) of the forebrain (Smart, 1961; Altman, 1969). From here they migrate into the olfactory bulb (OB) following a well defined pathway, the rostral migratory stream (RMS), which is oriented tangentially in relation to the ventricular and pia surfaces. Only in the $\mathrm{OB}$ do the cells reorient to reach their correct target layers and differentiate into GABAergic interneurons (Kishi, 1987; Luskin, 1993; Lois and AlvarezBuylla, 1994; for review, see O'Rourke, 1996; Alvarez-Buylla, 1997; Goldman and Luskin, 1998).

The mechanisms controlling the targeted translocation of large numbers of cells over a considerable distance $(>5 \mathrm{~mm}$ in the adult mouse) are an issue of intense investigation. Chain migration,

Received May 26, 1999; revised Nov. 29, 1999; accepted Dec. 1, 1999.

This work has been supported by institutional grants from the Centre National de la Recherche Scientifique and the European Community Biotech program to G.R. (BIO4-CT97-0329) and H.C. (BIO4-CT96-0730). We thank Geraldine Tetart, Gilberte Monti, and Jean-Paul Chauvin for technical help, Drs. Christo Goridis and Constantino Sotelo for support and discussion, and Dr. Patrick Carroll for helpful comments on this manuscript.

Correspondence should be addressed to Harold Cremer, IBDM, Campus de Luminy, Case 907, 13288 Marseille CEDEX 9, France. E-mail: cremer@ibdm. univ-mrs.fr.

Copyright (C) 2000 Society for Neuroscience $\quad 0270-6474 / 00 / 201446-12 \$ 15.00 / 0$ whereby the cells use each other as the migratory substrate, has been proposed to be the basis for the process, both for assembling the cells at the anterior horn of the lateral ventricle and also for the targeted migration within the RMS itself (Lois et al., 1996; Doetsch et al., 1997). Furthermore, continuous tunnels of glial cells, morphologically and molecularly distinct from radial glia, have been demonstrated in the pathway and proposed to play a role in the migratory process (Jankovski and Sotelo, 1996; Lois et al., 1996; Peretto et al., 1997).

A major question concerns the nature of the molecular cues involved in the correct targeting of the migrating precursors. The secretion of a chemoattractant factor by the OB appears as a possibility. Nevertheless, tissue derived from this structure had no directive influence on the migration (Hu and Rutishauser, 1996; Janovski et al., 1998). On the other hand, a septum-derived secreted factor showed a repulsive effect on the SVZ cells (Hu and Rutishauser, 1996). More recently, it has been shown that the secreted molecule slit shows such a repelling effect on SVZderived precursors (Wu et al., 1999). Furthermore, integrins have been demonstrated to have a regulatory influence on precursor cell chain-migration and regulation of their divisions (Jacques et al., 1998).

The highly polysialylated form of the neural cell adhesion molecule (NCAM) appears as another most promising candidate. Mice deficient for all splice variants (Cremer et al., 1994) or only the $180 \mathrm{kDa}$ isoform (Tomasiewicz et al., 1993) of NCAM show a dramatically size-reduced $\mathrm{OB}$ and an accumulation of migrating precursors along the RMS. In early postnatal stages this effect is phenocopied by enzymatic removal of polysialic acid (PSA) from 
the pathway (Ono et al., 1994), indicating the important role of the PSA modification in this process.

The consequences of PSA removal from the RMS by genetic or enzymatic methods have thus far been studied exclusively in early postnatal stages in mice lacking only the NCAM-180 isoform (Ono et al., 1994; Hu et al., 1996). However, tangential cell migration persists in the adult animal, and the well-defined and thin-diameter pathway in later stages appears strikingly different from that of young mice in which a massive wave of migration occurs (Kishi, 1987; Kishi et al., 1990; G. Chazal and H. Cremer, unpublished observations).

Here we present a detailed study of the consequences of NCAM deficiency (Cremer et al., 1994) on tangential cell migration in adult mice. In these mutants we find striking differences in the cellular organization and membrane contacts of the pathway, which might be responsible for severe alterations in cell migration. We also present evidence that the lack of PSA-NCAM interferes mainly with the organization of the stationary environment and its recognition by the migrating precursors and only marginally with the interactions between the precursor themselves. In vitro experiments, in which PSA was enzymatically removed from cultured SVZ explants, confirmed this observation. Finally, using transplantation experiments, we demonstrate that the mutant pathway is functional in the transport of neural precursors.

\section{MATERIALS AND METHODS}

Animals. NCAM-deficient mice have been described previously (Cremer et al., 1994). All analyses were performed on a C57BL/6J background (five backcrosses) in mice between 3 and 6 months of age.

Immunohistochemistry. For all histological analysis requiring perfusion, mice were deeply anesthetized with an overdose of xylazineketamine. Perfusion was performed intracardiacally with a solution of $4 \%$ paraformaldehyde in PBS. The brain was dissected out and immersed overnight in the same fixative at $4^{\circ} \mathrm{C}$. Coronal and sagittal sections were serially cut at $50 \mu \mathrm{m}$ using a Vibratome. Immunohistochemistry was performed on floating Vibratome sections, as described previously (Calaora et al., 1996; Cremer et al., 1998). Briefly, sections were first incubated overnight at $4{ }^{\circ} \mathrm{C}$ with the following antibodies: PSA (dilution 1:200; Rougon et al., 1986), mCD24 (dilution 1:100; Rougon et al., 1991), anti-GFAP antiserum (1:100, Sigma), and anti-neurofilament (SMI-31; dilution 1:2000; Sternberger Monoclonals) before incubation with the corresponding fluorescent-labeled secondary antibody. Controls were performed either by omitting the first antibody or by replacing the first specific antibody with a nonimmune serum. Sections were analyzed using a standard Leitz (Wetzlar, Germany) confocal microscope and the complementary software package. For three-dimensional reconstruction, 10 individual virtual sections were reconstructed.

BrdU injections and staining. A sterile solution of BrdU (Sigma) at 10 $\mathrm{mg} / \mathrm{ml}$ in PBS was injected intraperitoneally in mice at postnatal day 60 (P60) $(50 \mathrm{mg} / \mathrm{kg}$ of body weight). To label the entire pathway and for counts of dividing astrocytes, four injections were given over $2 \mathrm{~d}$; alternatively one injection was given $1 \mathrm{hr}$ before perfusion. Vibratome sections of the brains were processed for immunohistochemistry, as described above. To denature DNA, sections were treated for $30 \mathrm{~min}$ at $37^{\circ} \mathrm{C}$ with $\mathrm{HCl} 2 \mathrm{~N}$ in PBS containing $0.5 \%$ Triton $\mathrm{X}-100$, then rinsed three times in sodium tetraborate buffer $(0.1 \mathrm{M}, \mathrm{pH} 8.5)$ before incubation with anti-BrdU antibody (1:100; Dako, Glostrup, Denmark).

Conventional electron microscopy. For EM analysis adult mice were deeply anesthetized with an overdose of xylazine-ketamine and successively perfused with (1) $5 \mathrm{ml}$ of PBS, (2) $20 \mathrm{ml}$ of $4 \%$ paraformaldehyde (PFA) and $0.1 \%$ glutaraldehyde $(\mathrm{G})$ in $0.1 \mathrm{M}$ sodium phosphate buffer (pH 7.4), and (3) $10 \mathrm{ml}$ of the same mixture without glutaraldehyde but containing sodium- $m$-periodate and lysine. Fifty-micrometer-thick sagittal or transversal Vibratome sections were post-fixed for $1 \mathrm{hr}$ in osmium tetroxide. Dehydration was performed through increasing concentrations of ethanol followed by immersion in propylene oxide, propylene-Epon solution (1:1), and finally pure Epon. After infiltration overnight in Epon, the slices were flat-embedded between two plastic slides. Selected areas from the RMS were cut from the plastic wafer and semifine $(2 \mu \mathrm{m})$ or ultrathin $(70 \mathrm{~nm})$ sectioned. The sections were mounted on single-hole copper grids, stained with uranyl acetate and lead citrate, and examined with a Zeiss electron microscope.

Immunoelectron microscopy. Animals were perfused with a mixture of $4 \%$ PFA and $0.1 \%$ G. Vibratome sections $(300 \mu \mathrm{m})$ were fixed and embedded in LR Gold (TAAB Laboratories Equipment), as described in Berryman and Rodewald (1990). Ultrathin sections were incubated with anti-PSA IgG (1:500; Pasteur Mérieux) or anti-BrdU IgG for $2 \mathrm{hr}$ at RT and revealed with $10 \mathrm{~nm}$ gold-labeled goat anti-mouse $\operatorname{IgG}$ (1:30; Aurion).

For pre-embedding immunogold electron microscopy, Vibratome sections $(70 \mu \mathrm{m})$ were first treated with $\mathrm{HCl} 2 \mathrm{~N}$ to denature DNA, as described before, then incubated at $4^{\circ} \mathrm{C}$ during $24 \mathrm{hr}$ with anti-BrdU antibody (mouse IgG; dilution 1:100). They were post-fixed for $5 \mathrm{~min}$ in $4 \%$ PFA in PBS and incubated for $24 \mathrm{hr}$ at $4^{\circ} \mathrm{C}$ with the secondary $0.8 \mathrm{~nm}$ gold antibody (goat anti-mouse IgG; Aurion), diluted $1: 30$ in $0.1 \%$ fish gelatin. The next day, a silver enhancement was performed for $10 \mathrm{~min}$. The sections were osmicated, dehydrated, and flat-embedded in Epon resin. Ultrathin sections were performed on the selected areas and visualized with a Zeiss electron microscope.

SVZ explant culture. Cultures of SVZ explants were performed as described in Wichterle et al. (1997). Briefly, 5-d-old mice were anesthetized by hypothermia and then killed by rapid decapitation. Brains were dissected out and placed in ice-cold HBSS medium (Life Technologies). After Vibratome sectioning, the SVZ from the lateral wall of the anterior horn of the lateral ventricle was dissected out from the appropriate section and cut into pieces of 100-300 $\mu \mathrm{m}$ in diameter. The explants were mixed with Matrigel (Beckton Dickinson, Mountain View, CA) and cultured in four-well dishes. After polymerization for $10 \mathrm{~min}$, the gel was overlaid with $2 \mathrm{ml}$ of serum-free medium containing B-27 supplement (Life Technologies), in presence or absence of $70 \mathrm{U}$ of Endo $\mathrm{N}$ per milliliter prepared in our laboratory (Wang et al., 1994). Culture were maintained in a humidified, $5 \% \mathrm{CO}_{2}, 37^{\circ} \mathrm{C}$ incubator.

Cell migration distance. After $48 \mathrm{hr}$ in culture, explants were examined using phase-contrast microscopy (Axiovert 35M, Zeiss, Germany). Images were collected with a video camera (Cool View; Photonic Science) digitized, and analyzed using image-processing software (Visiolab1000; Biocom). Migration distance, calculated on three different experiments, including at least five explants per condition, was calculated as the distance in micrometers between the edge of the explant and the border of the cell migration front. Eight measurements were performed for each explant. The significance of the differences in cell migration under the two experimental conditions was calculated by Student's $t$ test.

Transplantation. Tissue isolated from the adult SVZ of the transgenic mouse line $\beta 2 \mathrm{nZ} 3^{\prime} 1$, expressing lacZ under the control of elements of the $\beta 2$-microglobulin promoter in subsets of developing and adult neurons (Cohen-Tannoudji et al., 1992), was stereotaxically grafted into the parenchyma neighboring the lateral ventricle of wild-type $(n=5)$ and NCAM-deficient $(n=5)$ mice, as described before (Jankovski and Sotelo, 1996), using the coordinates of Lois and Alvarez-Buylla (1994). After a survival time of $30 \mathrm{~d}$, animals were perfused and sectioned as described above. To detect $\beta$-galactosidase activity, the sections were incubated in an histochemical reaction containing 5-bromo-4-chloro-3indolyl- $\beta$-D-galactopyranoside (Xgal), a substrate that forms a blue precipitate, as described in Sotelo et al. (1994). Sections were mounted on slides and counterstained with neural red.

\section{RESULTS}

\section{Morphology of the RMS in NCAM-deficient mice}

Nissl staining of sagittal sections from adult wild-type mice revealed the presence of a continuous and defined pathway linking the anterior horn of the lateral ventricle to the ipsilateral olfactory bulb (Fig. 1a). The pathway was located between the overlying corpus callosum and the striatum in regions proximal to the ventricle; rostrally, it bordered the nucleus accumbens septi and the anterior olfactory nucleus before entering the OB. The stream was of small and relatively regular diameter, only at the most ventral part it appeared wider and fanned out.

In NCAM-deficient mice, the RMS also appeared as an unbroken connection between the lateral ventricle and the ipsilateral olfactory bulb (Fig. 1b). The pathway was located in the same 

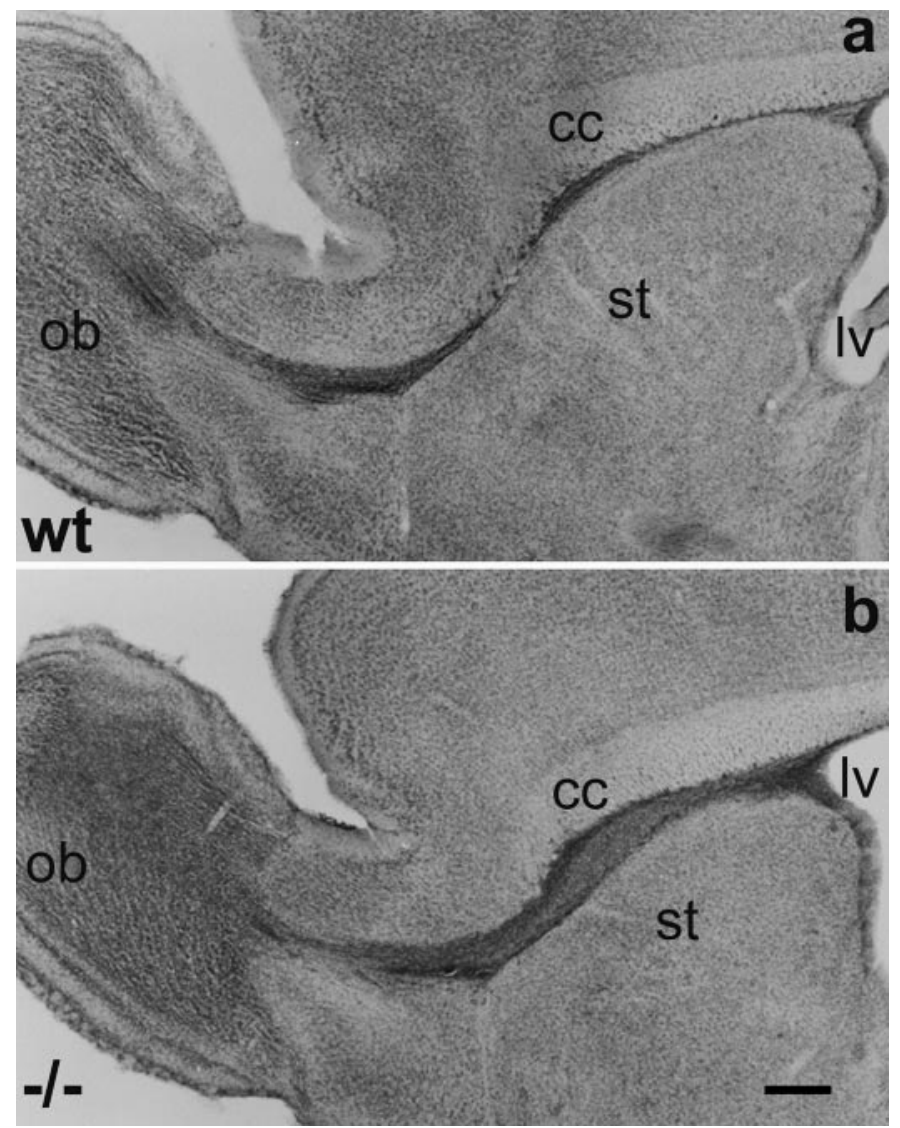

Figure 1. Localization of the RMS in adult wild-type $(a, w t)$ and NCAM-deficient $(b,-/-)$ mice. Light micrographs of Nissl-stained sagittal sections through the forebrain reveal in both animals a continuous pathway connecting the anterior horn of the lateral ventricle $(l v)$ to the center of the olfactory bulb $(o b)$. In the wild-type, the pathway is of small diameter over its entire length. Only at its most ventral part it appears slightly dispersed. In the mutant, the pathway is larger in diameter, especially in its caudal portion between the corpus callosum $(c c)$ and the striatum (st), but represents an unbroken and anatomically correct localized structure. Scale bar, $0.5 \mathrm{~mm}$.

position as in the wild-type and neighbored the same structures, but appeared larger in diameter over its entire length.

In the wild-type, PSA-NCAM, the most used marker for the RMS, was expressed along the entire pathway and revealed the chain-like organization of the structure (Fig. 2b; Rousselot et al., 1995). Immunogold staining for PSA at the ultrastructural level (Fig. 2e) demonstrated the expression of the determinant restricted to the membrane of neural precursors, thereby showing a high degree of clustering (Fig. 2e, arrows). Astrocytes were always devoid of staining (data not shown). To analyze the structure and composition of the mutant RMS in the absence of PSA-NCAM, we used antibodies against the glycosyl-phosphatidylinositol anchored glycoprotein mCD24 (Nedelec et al., 1992). During development and in adult stages, $\mathrm{mCD} 24$ was shown to be expressed in a pattern comparable to that of PSA-NCAM (Fig. $2 a$; Calaora et al., 1996). Confocal laser microscopy revealed in the wild-type the highly colocalized expression of the two molecules on the precursors arranged in chains (Fig. $2 a-c$ ). The pathway was of small diameter with the cells organized in chain-like aggregates oriented strictly in the direction of migration.

In the mutants, the mCD24 antibody also stained cells in the whole pathway while PSA staining was entirely lacking, as ex-
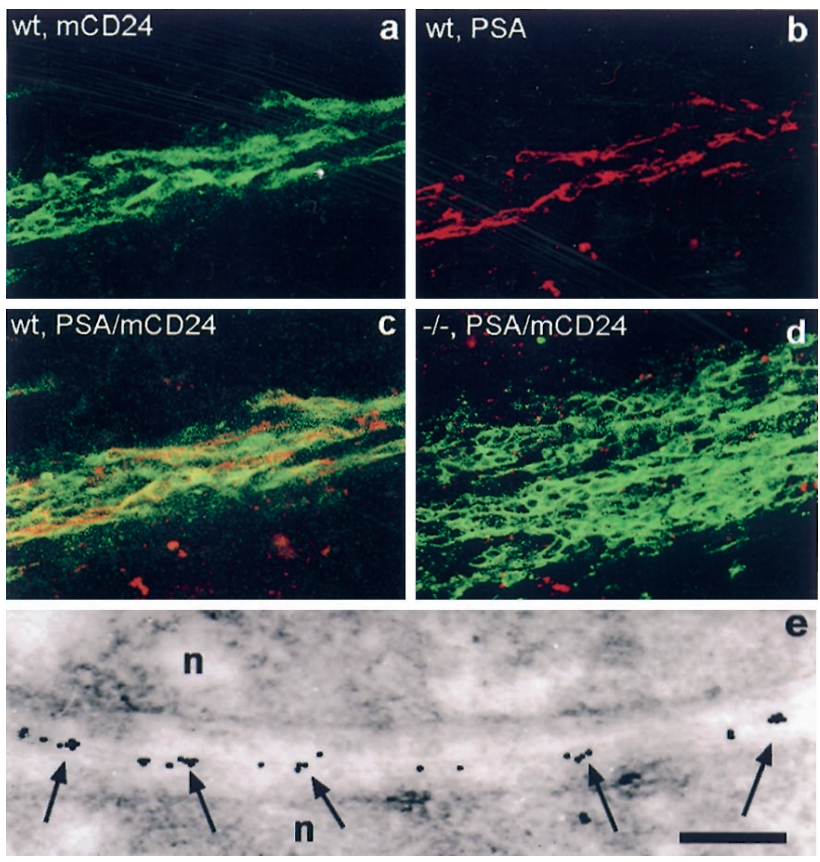

Figure 2. Expression of PSA-NCAM and mCD24 in the wild-type ( $a-c$, $e, w t)$ and NCAM-deficient $(d,-/-)$ RMS. In the wild type, confocal microscopy reveals that staining for $\operatorname{mCD} 24(a)$ and PSA-NCAM $(b)$ are largely overlapping $(c)$ and shows the chain-like arrangement of neural precursors migrating in the pathway. In mutants $(d)$, mCD24 labeling demonstrates the massive accumulation of cells in the RMS. Nevertheless, the chain-like organization is still obvious, whereas PSA-NCAM staining is totally absent, as expected. $e$, Immunoelectron microscopic image of the contact area of two neural precursors $(n)$ in the wild-type RMS at $20,000 \times$ magnification. PSA-associated gold particles are found in clusters (arrows) distributed over the membranes. Scale bar: $a-d, 50 \mu \mathrm{m} ; e, 300 \mathrm{~nm}$.

pected. However, the RMS was of considerably wider diameter, consistent with the alterations we found in the Nissl-stained material (Fig. $2 d$ ). Cells in the pathway were closely apposed and showed some degree of chain-like arrangement, although less pronounced than in the controls (Fig. 2d). As further PSAindependent markers for immature neurons we used antibodies against class III $\beta$-tubulin ( $\mathrm{TuJ} 1$ ) and nestin, which gave comparable results to $\mathrm{mCD} 24$ staining (data not shown).

\section{Arrangement of glia and axons}

Astrocytes, organized in tube-like aggregates, have been described in the RMS and proposed to be of importance for the migratory process in adults (Jankovski and Sotelo, 1996; Lois et al., 1996; Peretto et al., 1997). To investigate the consequences of NCAM deficiency on this cell population, we analyzed the expression of GFAP, a marker for a major subpopulation of astrocytes and known to be expressed in the RMS (Lois et al., 1996; Fig. 3). In the wild-type, prominent GFAP staining was visible from the SVZ to the OB, overlapping with the RMS, as revealed by Nissl staining (Fig. $3 a$ ). In addition, some positive cells were seen in the overlying corpus callosum, whereas the ventrally positioned striatum was devoid of GFAP immunoreactivity (Fig. $3 a$ ). Processes of these astrocytes were oriented rostrocaudally, ensheathing the mCD24-positive cell population and separating it from the surrounding parenchyma (Fig. $3 c$ ).

In the mutants, there was a massive increase in GFAP-positive structures along the RMS (Fig. 3b). In addition, the orientation of the glial processes in the direction of migration was less obvious, the processes being thicker and scattered over a wider area than 

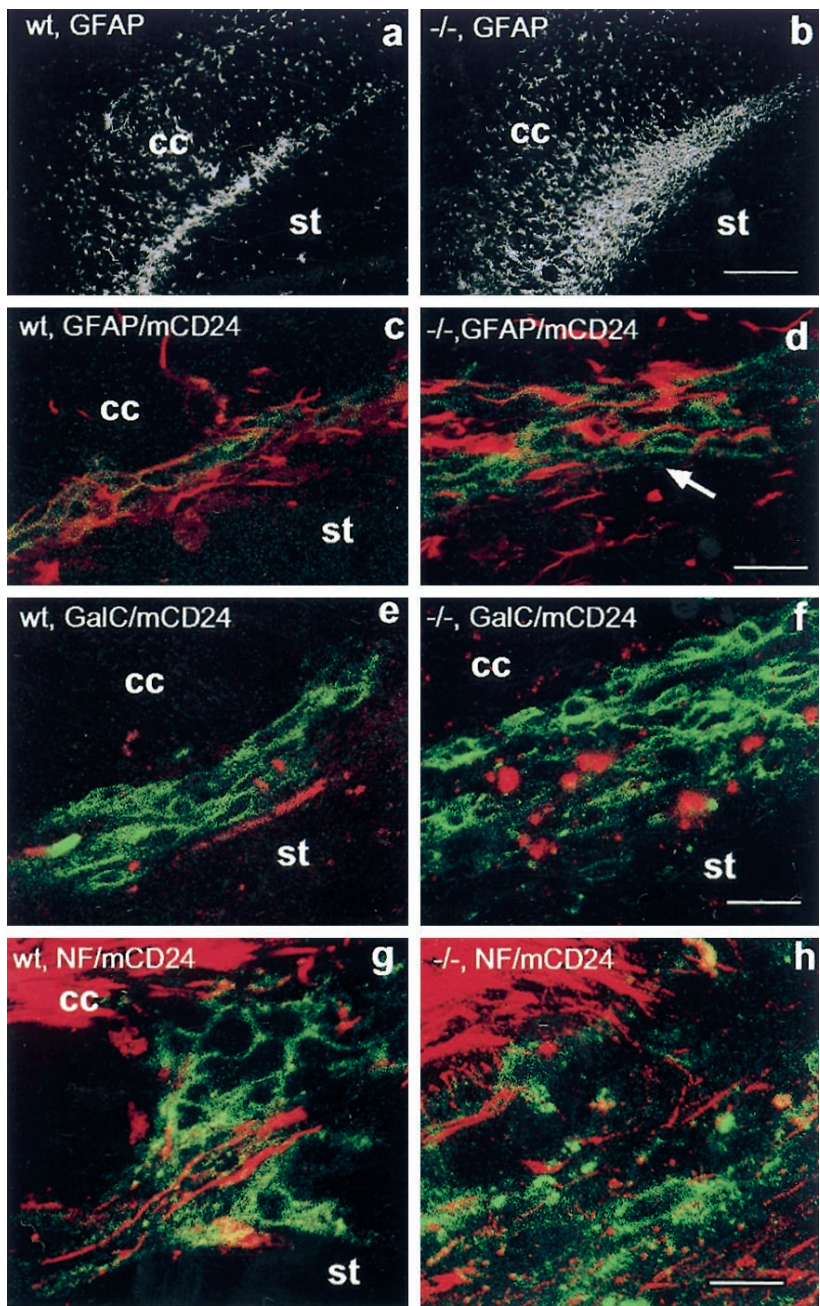

Figure 3. Arrangement of glia and axons in the wild-type $(a, c, g, w t)$ and NCAM-deficient $(b, d, h,-/-)$ RMS. Glial fibrillary acidic protein (GFAP) staining reveals the presence of astrocytes over the entire length of the pathway in wild-type $(a)$ and mutant animals $(b)$. In mutants, there is a massive accumulation of GFAP immunoreactivity distributed over a wider area. Double staining for GFAP (red) and mCD24 (green) demonstrates that in the wild-type $(c)$ glial processes, which are of small diameter and oriented in the direction of migration, ensheath and cover the entire free surface of chains. In the mutant $(d)$, GFAP immunoreactivity is strongly increased; the processes appear thicker in diameter and less organized in the rostrocaudal direction. In addition, the covering of precursors appears discontinuous, leaving them exposed to the environment (arrow). Labeling for the oligodendrocyte marker GalC $(e, f$, red; $\mathrm{mCD} 24$, green) reveals the presence of this cell type in the control $(e)$ as well as the mutant $(f)$ RMS. As for GFAP, GalC appears to be more expressed in the mutant pathway. Double staining for the axon marker neurofilament (NF, red) and mCD24 (green) demonstrates the presence of axons in the RMS. In the wild-type $(g)$, neurofilament positive structures are always well defined and oriented in the direction of migration, allowing the tracing of axon fascicles over considerable distances. In the mutant $(h)$, neurofilament immunoreactivity was much more abundant but dispersed, not showing the high degree of organization and orientation found in the wild-type. The punctuate aspect of the NF immunoreactivity indicates axons leaving the plane of the section. $c c$, Corpus callosum; st, striatum. Scale bars: $a, b, 400 \mu \mathrm{m} ; c-h, 30 \mu \mathrm{m}$.

in the wild-type (Fig. 3d). A large number of precursors were not ensheathed by glial processes and thus in direct contact with the surrounding tissue (Fig. 3d, arrow).

As a second glial cell type in the RMS, we identified oligodendrocytes using antibodies against GalC. As for GFAP, we found consistently increased immunoreactivity for this antigen (Fig. $3 e, f)$ in mutants.

As a further major component of the pathway, in addition to neural precursors and glial cells, we identified numerous axonal structures. In the wild-type, staining for the axonal marker neurofilament heavy chain revealed the presence of axon bundles between the chains of precursors identified by mCD24. These axons were oriented strictly in the direction of the migratory stream and could be followed over considerable distances in three-dimensional reconstructions of confocal images (Fig. $3 g$ ).

In the mutant RMS, neurofilament immunoreactivity was much more widespread. The staining was dispersed and did not show the high degree of organization and orientation found in the wild-type (Fig. 3h). Tracing of individual fascicles over long distances, as in the wild-type, was not possible. The appearances of extensive punctuate neurofilament staining suggested the presence of fibers leaving the plane of the sagittal section, thus oriented perpendicular to the direction of migration.

At the moment we can only speculate about the source of these axons. However, on some of our confocal three-dimensional reconstructions of neurofilament-stained sections, axons seem to invade the pathway from the surrounding tissue, as for example the striatum (Chazal and Cremer, unpublished observation). In contrast, we never saw any bundles emanating from the perpendicular running axons of the overlying corpus callosum.

\section{Ultrastructural organization}

Frontal sections through the wild-type RMS identified the typical organization of the pathway with its three major components: precursors, astrocytes, and axons (Fig. 4A,C). Neural precursors, distinguished by their more darkly stained electron dense nuclei of relatively regular shape, were always organized in groups with their membranes in close apposition, reflecting their organization in chain-like aggregates (Fig. $4 C$, small arrows). This arrangement was even more apparent in sagittal section (Fig. $5 A$ ) where chains of closely linked precursors could be traced over long distances (Fig. 5C, schematic representation). Astrocytes, identified by their more polymorph and lighter-stained nuclei and the presence of glycogen granules in their cytoplasm, were localized in the periphery of groups of progenitors (Figs. $4 C, 6 A$ ). Their processes ensheathed the chains of precursors (Figs. $4 C, 6 A$ ), thereby separating them completely from their environment, which consisted mainly of bundles of nonmyelinated and individual myelinated axons as well as blood vessels (Figs. 4A, $C, 5 A$ ). The equivalent diameter and shape of the axon profiles in transverse section indicated their strictly rostrocaudal orientation, parallel to the direction of migration (Figs. $4 C, 6 \mathrm{~A}$, large arrows).

In the mutant RMS, the general ultrastructural organization changed dramatically. The most obvious alteration was the massive accumulation of axonal structures (Fig. $4 B, D$ ), as was already suggested by the neurofilament staining (Fig. 3e,f). Although the relatively few axons present in the wild-type pathway show a high degree of fasciculation and a strictly rostrocaudal orientation (Figs. $4 C, 6 A$, large arrows), this configuration was almost entirely lost in the mutant (Figs. $4 B, D, 6 D$ ). Here, the axons in the RMS showed no obvious signs of bundling or specific orientation (Fig. $4 C, D)$. In addition, isolated precursors, not integrated into chains, became visible. Most of them were in direct contact with axonal structures lacking the separating glia sheath (Figs. $4 D, 5 B, 6 D$, arrow). The heterogeneous shapes of their nuclei, which in addition showed atypically deep invaginations (Fig. 4D), indicated a loss of rostrocaudal orientation. 

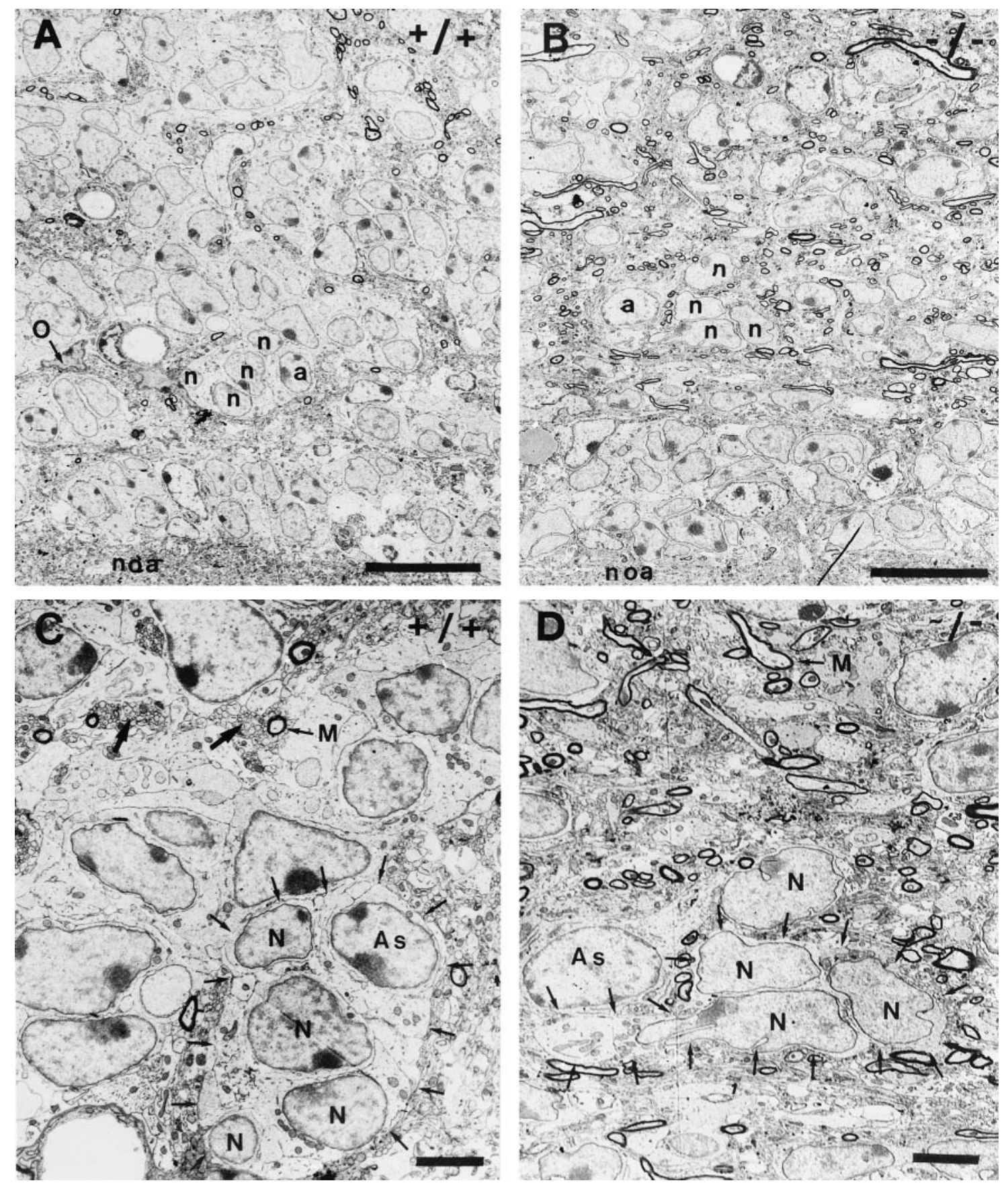

Figure 4. Ultrastructural organization of the wild-type $(A, C)$ and PSA-NCAM-deficient $(B, D)$ RMS. In frontal section, the wild-type RMS $(A)$ appears as a highly organized structure containing groups of neural precursors $(n)$ accompanied by astrocytes $(a)$. Individual oligodendrocytes $(O)$, axon profiles, and blood vessels were visible. The mutant pathway $(B)$ shows a lower degree of cell grouping and a massive invasion of axonal structures. In higher magnification $(C, D)$, it becomes obvious that in the wild-type $(C)$ groups of neural precursor $(N)$, representing chains (small arrows), are accompanied by astrocytes $(A s)$, together forming dense groups. Individual myelinated $(M)$ and bundles of nonmyelinated axons (large arrow) appear in proximity but are spatially separated from precursors. The equivalent diameter of these fibers shows the strictly parallel orientation, indicative of fasciculation. In contrast, the mutant pathway $(D)$ shows striking disorganization: a lower, but still considerable, degree of cell grouping (small arrows), astrocytes $($ As) separated from grouped precursors by intercalating axon profiles, and a massive invasion of these axonal structures. Note that these axons show no obvious signs of orientation or bundling. noa, Nucleus olfactorius anterior; Scale bars: $A, B, 10 \mu \mathrm{m} ; C, D, 3 \mu \mathrm{m}$.

Accompanying astrocytes, in the wild-type always in direct contact and ensheathing groups of precursors, were now isolated or only partially connected to these cells (Figs. $4 D, 6 D$ ). Although astrocytes were present, continuous tubes, as seen in the wildtype (Fig. 6A), could not be identified. Despite these drastic changes, the majority of precursors were still grouped together in closely linked clusters, suggestive of an arrangement in chains (Fig. $4 B, D$ ). This organization, reminiscent of the wild-type situation, was even more obvious in the sagittal plane, where chains could be followed over long distances, even in the absence of the ensheathing glia (Fig. 5B). Nevertheless, these were less frequent and more dispersed (Fig. 5C,D, compare schematic rep- 

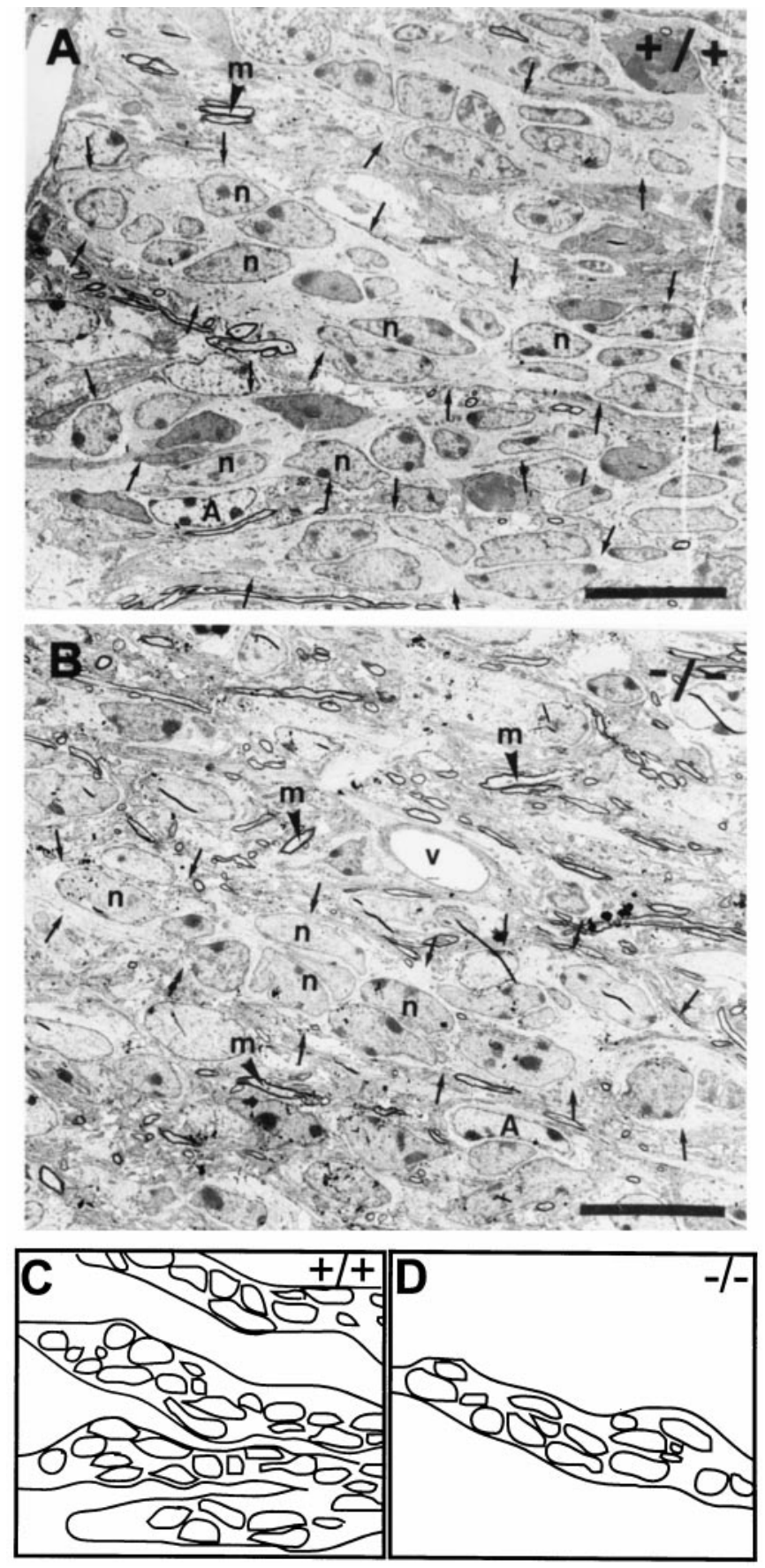

Figure 5. Organization of the RMS in sagittal section. In the wild-type $(A)$, the neural precursors $(n)$ show an elongated shape and are organized in continuous chains (small arrows). In the mutant $(B)$ neural precursors ( $n)$ appear more variable in shape, but the majority is still organized in rostrocaudally oriented chain-like arrangements, which can be traced over long distances $(C, D$, schematic representations). Nevertheless, chains are less frequent and more dispersed. The entire pathway in mutants appears highly disorganized because of the presence of many nonmyelinated and myelinated $(m)$ axons, which show no preferential orientation or organization. $A$, Astrocyte; $v$, blood vessel. Scale bar, $10 \mu \mathrm{m}$.

resentations). Instead, isolated precursors without obvious contact to aggregates were found.

The histological changes in the mutant RMS have been evaluated quantitatively via cell and axon counts on electron micrographs at different levels of the RMS (Table 1). A variety of morphological criteria have been used for the discrimination of the different cell types, as described (Jankovski and Sotelo, 1996; Doetsch et al., 1997). At all levels of the pathway, we found an approximately twofold increase in the astrocyte-precursor ratio, verifying quantitatively the massive GFAP staining visible at the light microscopic level (Fig. 3b,d). Furthermore, we found a more than fourfold increase in the number of myelinated axon profiles in the PSA-NCAM-deficient RMS. As mentioned above, this increase is associated with an augmentation in GalC immunoreactivity, and cell counts at the EM level suggested a small increase in the number of oligodendrocytes within the pathway (Table 1).

\section{Membrane contacts}

The plasma membrane of precursors organized in chains established two main classes of contacts: (1) those without obvious cytological specializations in the areas of contact, and (2) those presenting features of junctions. The first class of highly parallel membrane apposition was the most common, cells adhering to one another at any part of their surfaces (Fig. 6A). Less common but clearly visible were specialized junctions between the membranes of precursors, which we characterized as the zona adherens type. They presented flocculent material in the intracellular cleft and densely stained material on the cytoplasmic side of the membrane (Fig. 6B). Specialized contacts between glial cell processes were numerous. They were identified as typical gap junctions with a characteristic dense line between the associated membranes (Fig. 6C). Specialized contacts between precursors and glia cells were never observed.

In the mutant, in addition to the homotypic specialized contacts described above (Fig. 6E, $G$ ) zona adherens-like heterotypic precursor-astrocyte contacts were identified (Fig. 6F). Furthermore, high-magnification images demonstrated that the general membrane apposition of the different cell types in the pathway, which in the wild-type was characterized by a regular and parallel organization (Fig. 6A; Gregory et al., 1988), was different in the mutants. Here the membranes appeared much more irregular and curved (Fig. 6A,D, compare precursors).

\section{Precursors and glial cells are generated in the RMS}

Using the cell-division marker BrdU we analyzed the proliferating cell populations in the wild-type and NCAM-deficient RMS. Four injections were given over a period of $48 \mathrm{hr}$. In both mutants and wild-types, immunolabeling for the presence of incorporated BrdU revealed dividing cells at the SVZ and along the entire length of the pathway (Fig. 7a,b), overlapping almost perfectly with the RMS as identified by Nissl staining (compare Fig. 7a, $b$ to Fig. 1a,b).

To characterize the dividing cell populations in the RMS itself, we injected BrdU $1 \mathrm{hr}$ before perfusion and used double immunostaining for BrdU and PSA, mCD24, or GFAP. In control animals, double labeling for PSA and BrdU revealed the presence of dividing precursors integrated in chains along the entire pathway (Fig. $7 c, d$ ). The distribution and organization of these cells were equivalent to the BrdU/mCD24-positive population (Fig. $7 e$ ), again suggesting that $\mathrm{mCD} 24$ labeled the same group cells. Accordingly, we identified mCD24/BrdU-positive cells in the NCAM-deficient RMS (Fig. 7f). Furthermore, individual dividing astrocytes could be identified using GFAP/BrdU immunostaining in wild-type as well as mutant mice (Fig. 7g,h). To investigate if the massive over-representation of astrocytes in the mutant RMS is a consequence of their permanent overproduction, we aimed to quantify the amount of newly generated cells of 
Figure 6. Cell contacts in the wild-type $(A-C)$ and mutant $(D-G)$ RMS. In the wild-type $(A)$, groups of neural precursor $(N)$ are closely apposed and separated from axons (asterisks) and astrocytes $(A s)$, containing clusters of glycogen granules $(G)$. Contacts between precursor $(B)$ are of the zona adherens type with flocculent material in the intercellular cleft and stained material on the cytoplasmic side. Contacts between glial cells were characterized as typical gap junctions $(C)$. Heterotypic contacts between precursors and glia were never seen. In the mutant $(D)$, precursors can still be found as groups but appear less organized. Astrocytic processes $(A s)$ enwrap precursors only partially, leaving them exposed to surrounding parenchyma. Homotypic contacts $(\mathrm{N} / \mathrm{N} ; \mathrm{As} / \mathrm{As})$ in mutants were comparable to the wild-type $(E, G)$, but in addition many heterotypic precursor astrocyte membrane specializations of the zona adherens type are found $(F)$. Scale bars: $A, D, 1 \mu \mathrm{m} ; B, C, E, F, G, 200 \mathrm{~nm}$.
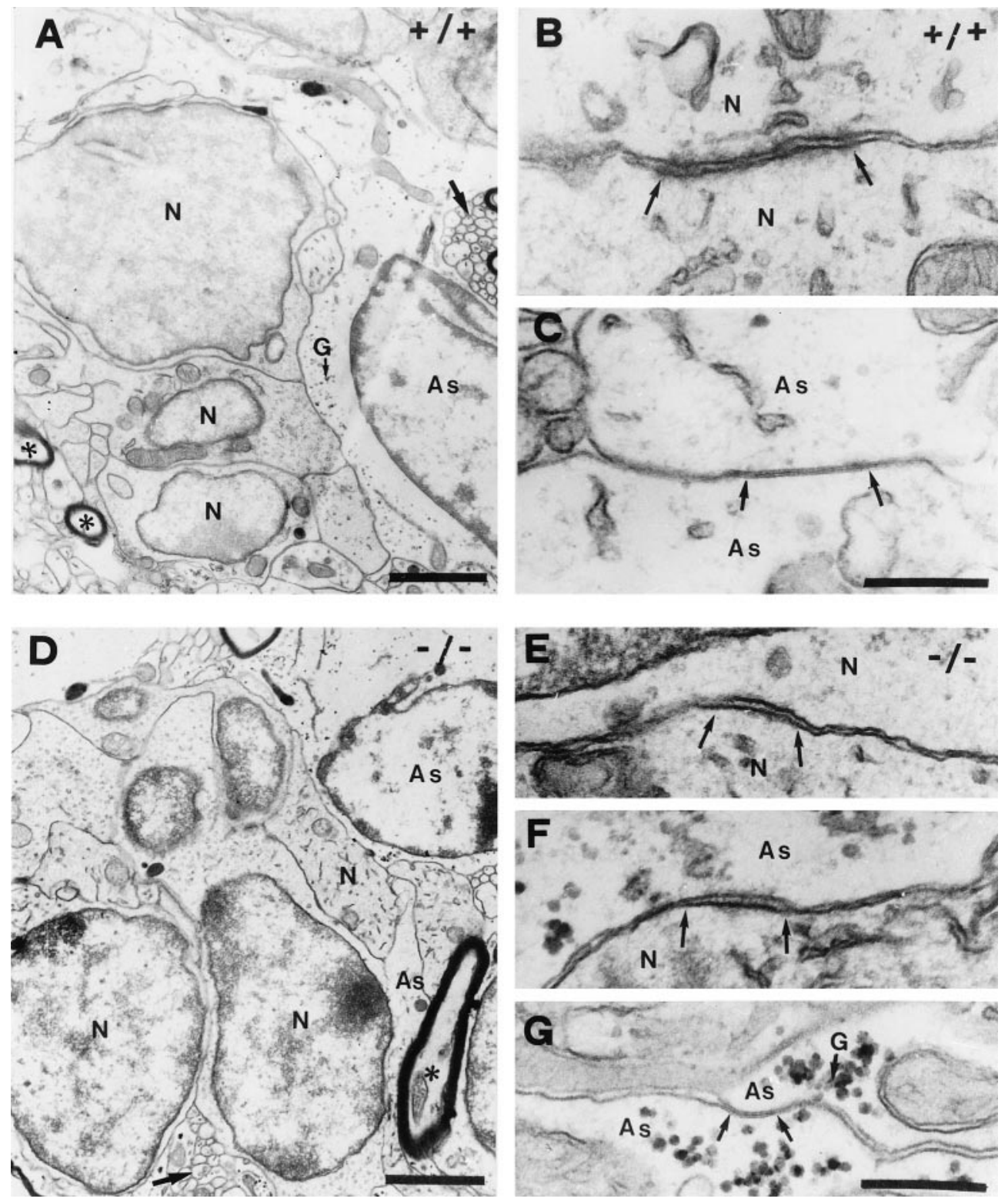

Table 1. Abundance of precursors, astrocytes, and axons in the wild-type and mutant RMS

\begin{tabular}{llc} 
NCAM & $+/+$ & $-1-$ \\
\hline Precursors & $85.2 \% \pm 1.7 \%(n=585)$ & $77.5 \% \pm 2.4 \%(n=399)$ \\
Astrocytes (total) & $14.8 \% \pm 1.5 \%(n=102)$ & $22.5 \% \pm 1.7 \%(n=116)$ \\
Astrocytes (BrdU+) & $4 \%(2 / 49)$ & $4.8 \%(6 / 126)$ \\
Oligodendrocytes & 4 & 7 \\
Myelinated axons & 131 & 571
\end{tabular}

Absolute numbers of precursors and astrocytes were identified and counted in semithin sections at three different levels (see schematic drawing, levels 1-3) of the RMS. Newly generated (BrdU+) astrocytes and oligodendrocytes, as well as myelinated axons were identified in ultrathin sections at levels 1 and 2, thus in more central parts of the pathway. this type. Immunogold labeling of BrdU-positive cells and analysis at the EM level turned out to be the only reliable way to doubtlessly identify and count this cell type (Fig. 7i,j). In reconstructions of comparable areas from two different regions of the RMS, we identified in the wild-type $4.1 \%$ ( 2 of 49 ) of all astrocytes positive for BrdU. In the mutant 4.8\% (6 of 126) of all astrocytes had gold particles over their nuclei, suggesting that the proliferation rate of this cell type is comparable in both situations.

\section{Effects of PSA-NCAM removal on migration of SVZ cells in vitro}

To further examine the role of PSA-NCAM on the migratory behavior of SVZ cells, we set up an in vitro assay, in which SVZ explants were cultured for $2 \mathrm{~d}$ in matrigel. This three-dimensional 



Figure 7. Proliferation in the RMS. BrdU immunohistochemistry on sagittal sections revealed the presence of dividing cells over the entire length of the wild-type $(a)$ and mutant $(b)$ RMS when BrdU was administered over $48 \mathrm{hr}$. Double labeling and confocal microscopy in the matrix allows, in contrast to collagen (Hu et al., 1996), to analyze chain migration in vitro (Wichterle et al., 1997).

After $48 \mathrm{hr}$ in culture, an extensive network formed around the explants under control conditions (Fig. $8 A$ ). In the presence of Endo-N in the culture medium, which completely removes PSA from the surface of the cells (Wang et al., 1994), network formation and cell migration out of the explants was reduced but nevertheless considerable (Fig. 8B). Statistical analysis showed that the average migration distance was reduced at $\sim 25 \%$ in the presence of EndoN $(83.4 \pm 3.3 \mu \mathrm{m})$ compared to controls (112.6 $\pm 4.3 \mu \mathrm{m}$; Fig. $8 C$, frequency distribution plot). Furthermore, we found that the organization of migrating cells differed in the two conditions. After $24 \mathrm{hr}$ in culture under control conditions (Fig. 8D), migrating SVZ cells appeared always arranged in chains, thereby showing a high degree of compaction (Wichterle et al., 1997). In contrast, after $24 \mathrm{hr}$ of culture in the presence of EndoN, most cells appeared only in loose contact to each other during their migration away from the explant. However, few chain-like arrangements were identified, which showed a lower degree of compaction than the controls (Fig. 8E).

When control cultures were observed after $48 \mathrm{hr}$ (Fig. $8 F$ ), almost all migrating SVZ cells were found integrated into well compacted chains. These were generally longer than those observed at $24 \mathrm{hr}$, but showed a similar morphology. In the presence of EndoN, a chain-like arrangement of SVZ cells was clearly visible but less frequent. Nevertheless, chains could be identified and showed a morphology comparable to the controls (Fig. 8G), except for a lower degree of compaction among individual precursors.

\section{The mutant RMS is functional}

We used transplantation studies to investigate if the NCAMdeficient RMS was functional and able to transport NCAMexpressing neural precursors from the SVZ to the OB. Tissue isolated from the SVZ of the transgenic mouse line $\beta 2 \mathrm{nZ3}{ }^{\prime} 1$, expressing lacZ in subsets of developing and adult neurons (Cohen-Tannoudji et al., 1992), was grafted into the SVZ of wild-type $(n=4)$ and NCAM-deficient $(n=4)$ mice. Thirty days after the graft, animals were perfused and stained for the presence of $\beta$-galactosidase. In both groups only two of the transplanted animals showed $\beta$-galactosidase-positive cells integrated into the host tissue. In one of migration-positive mutant animals the graft was misplaced into the ventricle, but in close contact to the anterior wall. This was probably because of the larger size of the lateral ventricle in NCAM-deficient mice (Wood et al., 1998). In the other four animals, which showed no migration, the grafted tissue was not placed in the SVZ or in contact to the anterior wall of the ventricle.

In both control and mutant grafted mice, we found $\beta$-galactosidase-expressing cells integrated in the SVZ (Fig. 9a, $b$ ),

wild-type demonstrated the presence of PSA ( green)/BrdU (red) doublepositive cells integrated into chains $1 \mathrm{hr}$ after $\operatorname{BrdU}$ injection $(c, d)$. In the wild-type and mutant, mCD24 ( green)/BrdU (red)-labeled cells were comparably located within chain-like structures $(e, f)$. The RMS of both groups contained also individual GFAP ( green)/BrdU ( red)-stained cells, demonstrating the production of new astrocytes in the pathway. Both cell types, dividing neural precursors $(n)$ and astrocytes $(a)$ were also identified in the wild-type $(i)$ and mutants $(j)$ using immunogold labeling for BrdU and electron microscopy. Arrows indicate BrdU-associated gold particles overlying nuclei of dividing cells. Scale bar: $a, b, 1 \mathrm{~mm} ; c-h, 10$ $\mu \mathrm{m} ; i, j, 4 \mu \mathrm{m}$. 


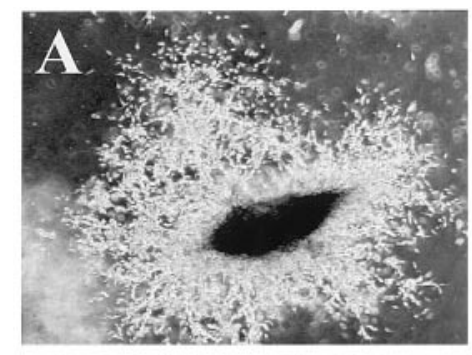

Figure 8. Cell migration from SVZ explants cultured in Matrigel. SVZ tissue was cultured in the absence $(A, D$, $F)$ or presence $(B, E, G)$ of EndoN. Phase-contrast image of typical explants cultured in absence $(A)$ or presence $(B)$ of EndoN as has been used for the migration distance analyses. Note at this low magnification the more pronounced development of chains in the control $(A)$ compared to the EndoN-treated culture $(B) . C, \mathrm{Cu}$ mulative frequency distribution plot of the distance of SVZ cell migration in the absence (circles) or presence (squares) of EndoN. The values represent pooled data from three independent experiments. Aspect of migrating cells in absence of EndoN after $24(D)$ or $48(F)$ hr of culture. Note the compacted appearance of the cells integrated in chains at both time points. Borders between individual cells were not prominent ( $D, F$, black arrows). In the presence of EndoN for $24 \mathrm{hr}$, fully developed chains were rare (compare $A, B)$, but clearly distinguishable when they appeared $(E)$. In these aggregates individual cells (arrow) were easily distinguishable, contrary to the wildtype. After $48 \mathrm{hr}(G)$ well-defined aggregates became visible in the EndoNtreated cultures. Nevertheless, as at 24 $\mathrm{hr}$, borders between cells were more prominent, allowing the identification of individual cells ( $F, G$, compare black arrows).

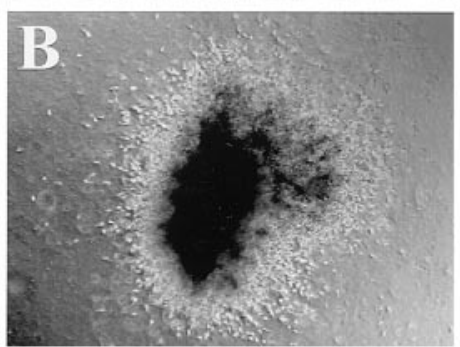

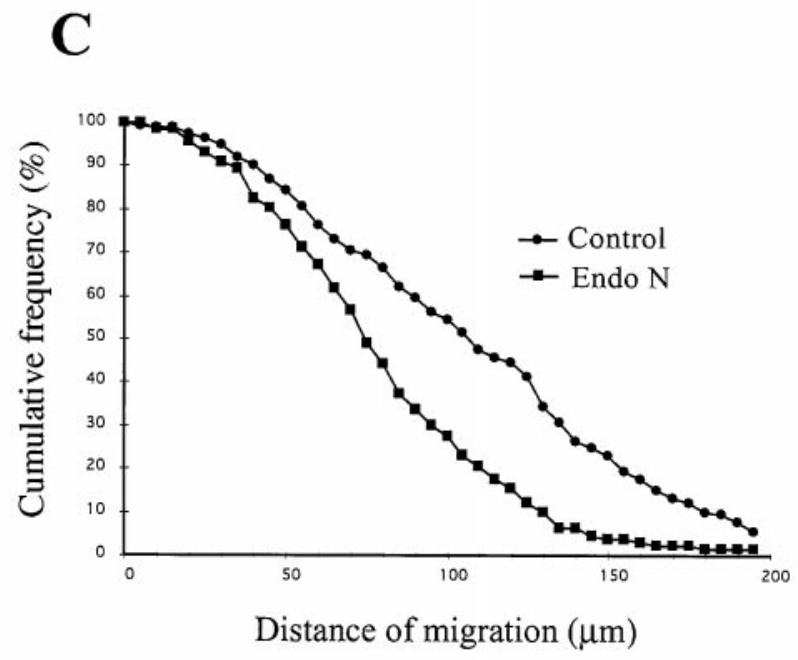
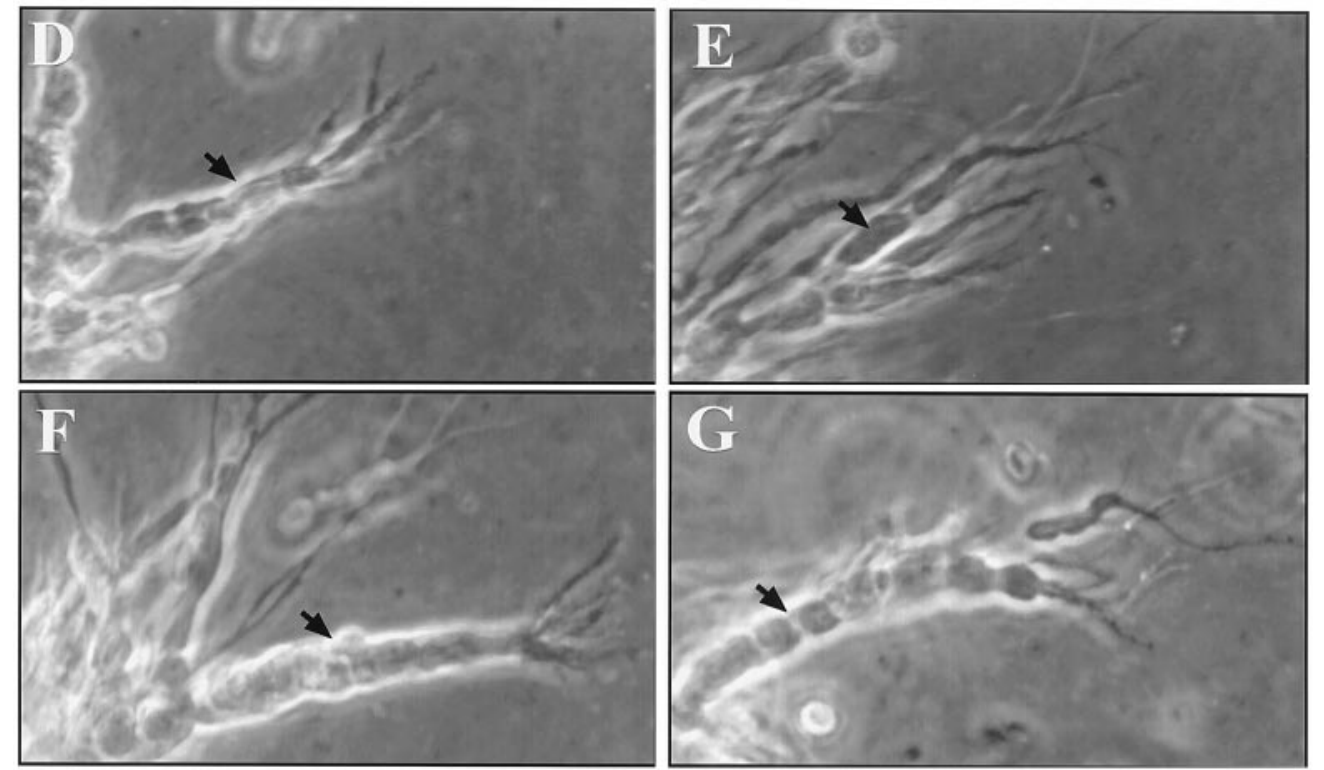

the RMS (Fig. 9c,d), and also in the OB (Fig. 9e,f). In controls, we found 22 of 164 lacZ-expressing cells integrated in the OB (13\%), whereas in mutants 6 of 106 labeled cells $(6 \%)$ reached their target. Although the low numbers of labeled cells do not permit a quantitative evaluation, these data clearly show that migration of PSA-NCAM-deficient neural precursors in a wild-type environment occurs.

\section{DISCUSSION}

In this study we investigated the structure and properties of the RMS in adult mice deficient for all NCAM isoforms, which have been shown to be also devoid of PSA (Cremer et al., 1994). Our main findings are that (1) a morphologically altered but defined pathway connects the SVZ to the OB in mutants, (2) mutant neural precursors are still able to form chains in vivo as well as in vitro, (3) the migratory environment, consisting of glial cells and axons, shows dramatic quantitative and qualitative changes, (4) membrane specializations, which in the wild-type occur only homotypically, are in the mutant additionally formed in a heterotypic manner, linking migrating precursors to glial cells, and (5) the PSA-NCAM-deficient pathway is able to transport precursors from the SVZ into the OB.

Neural precursors migrating in the RMS have been shown to be organized in particular chain-like arrangements, and chain migration, with the cells using each other as the migratory substrate, has been proposed to underlie the massive transport of cells in this system (Rousselot et al., 1995; Doetsch and Alvarez-Buylla, 1996; Lois et al., 1996; Wichterle et al., 1997). A function for PSA-NCAM in the formation of these chains has been suggested based on the expression of PSA-NCAM by the migrating cells (Rousselot et al., 1995; Wichterle et al., 1997) and the observation that migration in the RMS is hampered in mice lacking NCAM and PSA (Tomasiewicz et al., 1993; Cremer et al., 1994). Nevertheless, an active role for PSA-NCAM in the formation of chains has not been conclusively demonstrated so far.

We find that in all regions of the mutant RMS precursors are still able to form chain-like structures, although less frequent than in the wild type. Aggregates of closely linked cells could be traced over considerable distances in confocal and electron microscopic 

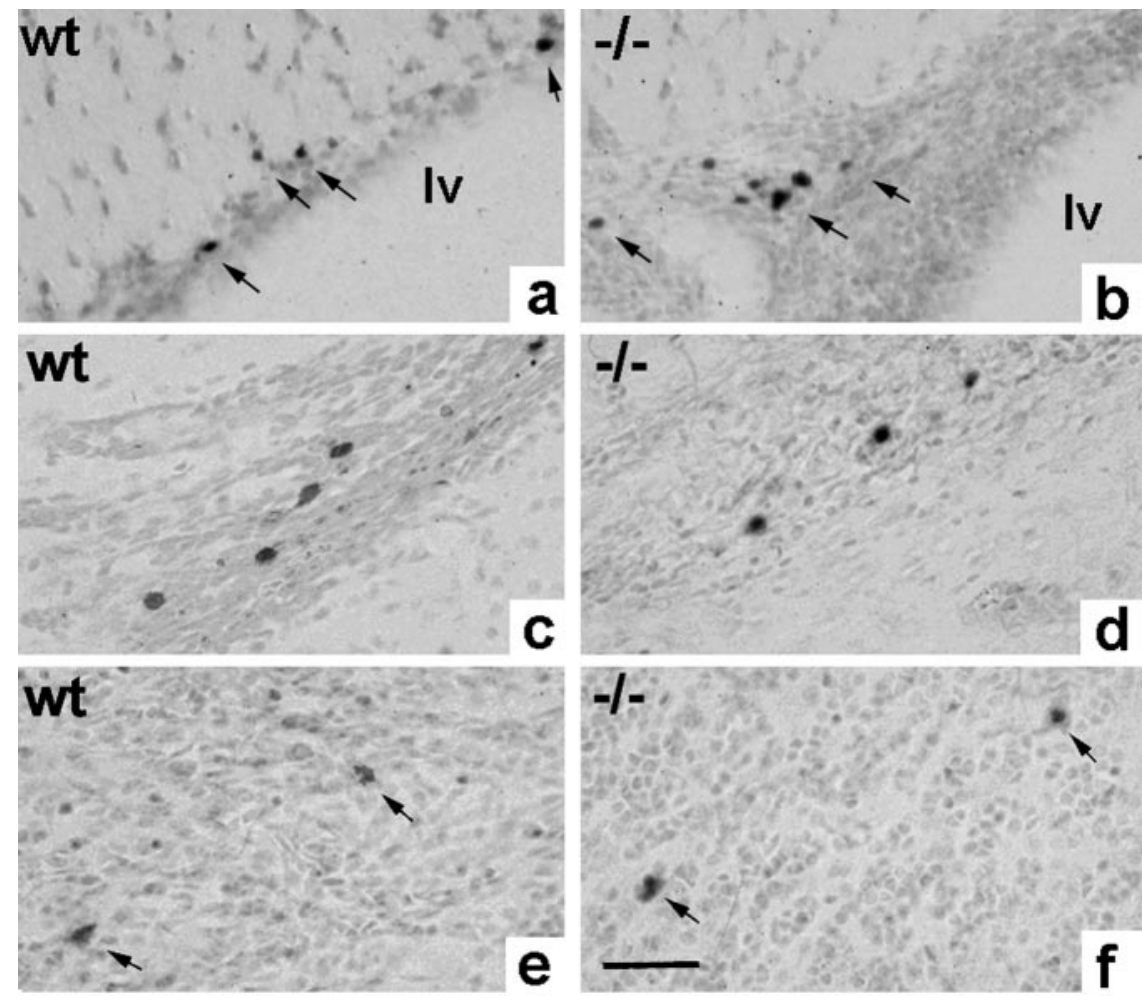

Figure 9. Functional properties of the PSA-NCAMdeficient RMS. PSA-NCAM-expressing cells, isolated from the SVZ of LacZ-expressing adult mice, were transplanted into the anterior horn of the lateral ventricle of wild-type and mutant mice. After a survival time of $30 \mathrm{~d}$ Xgal-positive cells were found integrated in the $\operatorname{SVZ}(a, b)$, the RMS $(c, d)$, and the $\mathrm{OB}(e, f)$ in both groups of animals. Scale bar, $30 \mu \mathrm{m}$. studies. This strongly suggests that undisrupted chains of neural precursors are able to form and transport cells in the absence of PSA-NCAM. In support, we find that the particular zona adherens-like membrane specializations, which have been shown to connect the migrating precursors to each other in the wild-type (Jankovski and Sotelo, 1996; Lois et al., 1996), still exist in the mutant RMS. We also find that after removal of PSA from SVZ explants in vitro distinguishable and well-defined chains develop. Although the formation of these aggregates appears delayed and show a lower degree of compaction than in the controls, this is also in favor of the idea that the basic ability of SVZ-derived neural precursors to form chains is largely independent of the presence of PSA-NCAM. Our findings are in agreement with results from transplantation experiments performed by $\mathrm{Hu}$ et al. (1996), showing that SVZ cells from NCAM-180-deficient mice transplanted into a wild-type RMS were able to migrate into the OB. The authors, however, argue that migration of these transplanted cells results from their integration in actively moving PSA-NCAM-expressing chains. We demonstrate here that mutant precursors are able to assemble and migrate in aggregates consisting entirely of PSA-NCAM-deficient cells in vivo as well as in vitro.

The question then occurred of whether the highly perturbed stationary environment in the mutants hampers migration between the SVZ and the RMS. We used transplantation of $\beta$-galexpressing wild-type cells into mutant and control brains to investigate this point. Thirty days after grafting into the lateral ventricle, $\beta$-gal-positive cells were found in the SVZ, RMS, and OB of mutant animals, clearly demonstrating that the mutant RMS is capable to transport cells over its entire length.

However, the finding that lacZ-expressing SVZ cells grafted into the adult mutant brain were able to migrate and reach the $\mathrm{OB}$ appears at first sight in striking contrast to the study of $\mathrm{Hu}$ et al. (1996). They reported that DiI-labeled wild-type SVZ tissue grafted into NCAM-180-deficient mice resulted in the almost complete absence of migration. A possible reason for this difference could be that in the first study early postnatal donor and host animals were used, whereas adult animals were investigated in this study. Migration in the early postnatal RMS might be more dependent on the presence of PSA-NCAM than in the mature structure. Alternatively, and in our opinion more likely, the discrepancy results from differences in the time of observation. Presumed that the rate of translocation is slower in the perturbed mutant RMS than in the wild-type, a $5 \mathrm{~d}$ delay before examination (Hu et al., 1996) could be insufficient to observe migration, whereas $30 \mathrm{~d}$ after grafting (our study), the cells have been able to reach the OB. This second explanation is also supported by our explant studies, performed with tissue from 5-d-old animals; thus comparable to the time window used by $\mathrm{Hu}$ et al. (1996). Our data clearly indicate that precursors from the early postnatal SVZ are still able to perform chain migration in matrigel, even after removal of PSA. The observation that generation of chains and their migration are delayed compared to the control situation, as suggested by these in vitro results, might well account for the differences in the outcome of the two studies. However, a passive "towing along" of isolated mutant precursors by PSA-expressing chains, a mechanism proposed by $\mathrm{Hu}$ et al. (1996), appears not necessary to explain the migration of mutant SVZ cells in a wild-type environment.

Altogether, the presence of intact chains and the results of the transplantation studies are in favor of a more important role of PSA-NCAM in interactions of precursors with their environment, than between the migrating precursors themselves. The major component of the surrounding parenchyma are GFAPpositive astrocytes, which in the adult wild-type form continuous, tunnel-like sheaths and separate the chains of migrating neuronal cells completely from their environment (Jankovski and Sotelo, 1996; Lois et al., 1996; Peretto et al., 1997; this study). Because of 
the abundance and high degree of organization along the entire RMS, a function in the guidance of tangential migration has been proposed (Lois et al., 1996; Peretto et al., 1997). However, in vitro analysis revealed that the migration process itself is independent of glial cells (Wichterle et al., 1997; our studies).

The striking loss of the tube-like arrangement of astrocytes, leaving precursors directly exposed to the nearby parenchyma, represents probably the most severe morphological alteration in the mutant RMS. In addition to this general disorganization, we find a massive accumulation of astrocytes in the pathway, shifting the ratio between precursors and glial cells from 5.7:1 in the wild-type to $2.8: 1$ in the mutant. The finding that newly generated astrocytes in the pathway appear at about the same frequency in mutants and controls suggests that this over-representation is a consequence of a developmental accumulation and not attributable to a permanent massive increase in their proliferation rate. A further striking change is, that in the wild-type precursors form specialized membrane junctions only among themselves, whereas glial cells are homotypically connected by gap junctions (Jankovski and Sotelo, 1996; Lois et al., 1996). In mutants, we find in addition to these contacts a variety of precursors linked to astrocytes via zona adherens type junctions.

Thus, lack of NCAM results in a perturbation of neuron-glia interactions, and modifications in these interactions might in turn be responsible for the inhibition of migration in the RMS. It has been demonstrated that a cross-talk exists between neurons and glial cells and data in favor of an active role of PSA-NCAM in this process has been presented (Garcia-Segura et al., 1995; Loudes et al., 1997). The lack of PSA-NCAM on the surface of migrating precursors might alter the proliferative properties of this glial cell population, a scenario that appears reminiscent of astrogliosis occurring in neurodegenerative diseases even before any signs of neuronal damage (Hoffmann et al., 1992). It appears also conceivable that glial cells might perform the general function to enwrap any free membrane of migrating neural precursors. The existence of precursors not integrated in chains in mutants would represent additional membrane surface to cover and consequently be the reason for changes in the astrocyte/ precursor ratio.

As further components of the RMS, besides precursors and astrocytes, we identified bundles of nonmyelinated, individual myelinated axons as well as small amounts of oligodendrocytes. In the wild-type, the axons were generally oriented parallel to the direction of migration and could be traced over considerable distances within the pathway. Neurofilament staining suggested that they invade the pathway from the surrounding tissue, as for example the striatum. In contrast, we never saw any bundles emanating from the perpendicular running axons of the overlying corpus callosum (Chazal and Cremer, unpublished observation).

Axons in the RMS have been described before (Kishi et al., 1990; Jankovski and Sotelo, 1996), but a direct role in the migratory process has been excluded (Alvarez-Buylla, 1997) mainly for two reasons. First, most of the precursor somata are not in direct contact with axons (Kishi et al., 1990; this study), and second, DiI labeling studies did not reveal continuous fibers connecting the SVZ to the OB (Lois et al., 1996). Nevertheless, the fact that in the mutant RMS we observed a massive accumulation of axon profiles, myelinated processes being five times more abundant, as well as their striking loss of fasciculation, might reflect an active participation in the migration process. Such a function has already been shown for young tectobulbar neurons (Gray and Sanes, 1991) as well as migrating oligodendrocyte precursors
(Ono et al., 1997). However, a direct axonophilic mode of guidance (Rakic, 1990) in this system appears unlikely because, as already mentioned, most of the precursors are physically separated from axon bundles by glial cells. Thus, it appears more probable that the highly oriented fascicles, possibly originating from surrounding brain structures as for example the striatum, in concert with the tube-like arranged glial cells, provide the organized and permissive environment, which appears essential for the transport of large amounts of cells through the mature forebrain. In mutants, it seems possible that the considerably wider pathway, containing cells that migrate more individualized and show a lower degree of orientation, recruits more axons from the surrounding structures, leading to their striking overrepresentation. Furthermore, the disorganization of the migration process itself could account for the defasciculated appearance and loss of orientation of the axons. Alternatively, the defasciculation of axons in the mutant pathway might be a primary defect occurring during development. Evidence from our (Cremer et al., 1997) and other (Seki and Rutishauser, 1998) laboratories suggests, that lack of PSA-NCAM results in defasciculation of hippocampal mossy fibers during development. Because PSA-NCAM is expressed on all growing fiber tracts in the developing CNS, the perturbation effects seen in the hippocampus are likely to be relevant in other situations.

NCAM-deficient mice represent the first, and so far also the only, genetic model to study the in vivo functions of NCAM and PSA. Nevertheless, they are also charged with a variety of disadvantages. First, the fact that these animals are chronically devoid of NCAM does not allow discriminating, if the defects we find are consequences of alterations that occurred during development or if they are caused by the acute lack of the molecule in the adult. A second drawback lies in the fact that NCAM is the only known carrier for PSA, which makes it impossible to discriminate if phenotypic changes in NCAM-deficient mice are attributable to the lack of the protein itself or merely caused by the absence of PSA. Specific conditional mutants, lacking NCAM only at certain stages, or mouse models lacking either PSA or NCAM will be necessary to approach these questions.

\section{REFERENCES}

Alvarez-Buylla A (1997) Mechanism of migration of olfactory bulb interneurons. Cell Dev Biol 8:207-213.

Altman J (1969) Autoradiographic and histological studies of postnatal neurogenesis. IV. Cell proliferation and migration in the anterior forebrain with special reference to persisting neurogenesis in the olfactory bulb. J Comp Neurol 137:433-458.

Berryman MA, Rodewald RD (1990) An enhanced method for postembedding immunocytochemical staining which preserves cell membranes. J Histochem Cytochem 38:159-170.

Calaora V, Chazal G, Nielsen PJ, Rougon G, Moreau H (1996) mCD24 expression in the developing mouse brain and in zones of secondary neurogenesis in the adult. Neuroscience 73:581-594.

Cohen-Tannoudji M, Morello D, Babinet C (1992) Unexpected position-dependence of $\mathrm{H}-2$ and $\beta 2$ microglobulin/lacZ transgenes. Mol Reprod Dev 33:149-159.

Cremer H, Lange R, Christoph A, Plomann M, Vopper G, Roes J, Brown R, Baldwin S, Kraemer P, Scheff S, Barthels D, Rajewsky K, Wille W (1994) Inactivation of the N-CAM gene in mice results in size reduction of the olfactory bulb and deficits in spatial learning. Nature 367:455-459.

Cremer H, Chazal G, Goridis C, Represa A (1997) PSA-NCAM is essential for axonal growth and fasciculation in the hippocampus. Mol Cell Neurosci 8:323-335.

Cremer H, Chazal G, Carleton A, Goridis C, Vincent JD, Lledo PM (1998) Long-term but not short-term plasticity at mossy fiber synapses is impaired in neural cell adhesion molecule-deficient mice. Proc Natl Acad Sci USA 95:13242-13247. 
Doetsch F, Alvarez-Buylla A (1996) Network of tangential pathways for neuronal migration in adult mammalian brain. Proc Natl Acad Sci USA 93:14895-14900.

Doetsch F, Garcia-Verdugo JM, Alvarez-Buylla A (1997) Cellular composition and three-dimensional organization of the subventricular germinal zone in the adult mammalian brain. J Neurosci 17:5046-5061.

Garcia-Segura LM, Canas B, Parducz A, Rougon G, Theodosis D, Naftolin F, Torres-Aleman I (1995) Estradiol promotion of changes in the morphology of astroglia growing in culture depends on the expression of polysialic acid of neural membranes. Glia 13:209-216.

Goldman SA, Luskin MB (1998) Strategies utilized by migrating neurons of the postnatal vertebrate forebrain. Trends Neurosci 21:107-114.

Gray GE, Sanes JR (1991) Migratory paths and phenotypic choices of clonally related cells in the avian optic tectum. Neuron 6:211-225.

Gregory WA, Edmondson JC, Hatten ME, Mason CA (1988) Cytology of neuron glial apposition of migrating cerebellar granule cells in vitro. J Neurosci 8:1728-1738.

Hoffmann MC, Nitsch C, Scotti AL, Reinhard E, Monard D (1992) The prolonged presence of glia-derived nexin, an endogenous protease inhibitor, in the hippocampus after ischemia-induced delayed neuronal death. Neuroscience 49:397-408.

$\mathrm{Hu} \mathrm{H}$, Rutishauser U (1996) A septum-derived chemorepulsive factor for migrating olfactory interneuron precursors. Neuron 16:933-940.

Hu H, Tomasiewicz H, Magnuson T, Rutishauser U (1996) The role of polysialic acid in migration of olfactory bulb interneuron precursors in the subventricular zone. Neuron 16:735-743.

Jacques TS, Relvas JB, Nishimura S, Pytela R, Edwards GM, Streuli CH, ffrench-Constant C (1998) Neural precursor cell chain migration and division are regulated through different beta1 integrins. Development 125:3167-3177.

Jankovski A, Sotelo C (1996) Subventricular zone-olfactory bulb migratory pathway in the adult mouse: cellular composition and specificity as determined by heterochronic and heterotopic transplantation. J Comp Neurol 371:376-396.

Jankovski A, Garcia C, Soriano E, Sotelo C (1998) Proliferation, migration and differentiation of neuronal progenitors in the adult mouse subventricular zone surgically separated from its olfactory bulb. Eur J Neurosci 10:3835-3868.

Kishi K (1987) Golgi studies on the development of granule cells of the rat olfactory bulb with references to migration in the subependymal layer. J Comp Neurol 258:112-124.

Kishi K, Peng JY, Kakuta S, Murakami K, Kuroda M, Yokota S, Hayakawa S, Kuge T, Asayama T (1990) Migration of bipolar subependymal cells, precursors of the granule cells of the olfactory bulb, with reference to the arrangement of the radial glial fibers. Arch Histol Cytol 53:219-226.

Lois C, Alvarez-Buylla A (1994) Long-distance neuronal migration in the adult mammalian brain. Science 264:1145-1148.

Lois C, Garcia-Verdugo JM, Alvarez-Buylla A (1996) Chain migration of neuronal precursors. Science 271:978-981.

Loudes C, Rougon G, Kordon C, Faivre-Bauman A (1997) Polysialylated neural cell adhesion is involved in target-induced morphological differentiation of arcuate dopaminergic neurons. Eur J Neurosci 9:2323-2333.

Luskin MB (1993) Restricted proliferation and migration of postnatally generated neurons derived from the forebrain subventricular zone. Neuron 11:173-189.

Nedelec J, Pierres M, Moreau H, Barbet J, Naquet P, Faivre-Sarrailh C, Rougon G (1992) Isolation and characterization of a novel glycosylphosphatidylinositol-anchored glycoconjugate expressed by developing neurons. Eur J Biochem 203:433-442.

Ono K, Tomasiewicz H, Magnuson T, Rutishauser U (1994) N-CAM mutation inhibits tangential neuronal migration and is phenocopied by enzymatic removal of polysialic acid. Neuron 13:395-609.

Ono K, Yasui Y, Rutishauser U, Miller RH (1997) Focal ventricular origin and migration of oligodendrocyte precursors into the chick optic nerve. Neuron 19:283-292.

O'Rourke NA (1996) Neuronal chain gangs: homotypic contacts support migration in the olfactory bulb. Neuron 16:1061-1064.

Peretto P, Merighi A, Fasolot A, Bonfanti L (1997) Glial tubes in the rostral migratory stream of the adult rat. Brain Res Bull 42:9-21.

Rakic P (1990) Principles of neural cell migration. Experientia 46:882-891.

Rougon G, Dubois C, Buckley N, Magnani JL, Zollinger W (1986) A monoclonal antibody against meningococcus group $\mathrm{B}$ polysaccharides distinguishes embryonic from adult N-CAM. J Cell Biol 103:2429-2437.

Rougon G, Alterman LA, Dennis K, Guo XJ, Kinnon C (1991) The murine heat-stable antigen: a differentiation antigen expressed in both the hematolymphoid and cell lineages. Eur J Immunol 21:1397-1402.

Rousselot P, Lois C, Alvarez-Buylla A (1995) Embryonic (PSA) $\mathrm{N}$-CAM reveals chains of migrating neuroblasts between the lateral ventricle and the olfactory bulb of adult mice. J Comp Neurol 351:51-61.

Seki T, Rutishauser U (1998) Removal of polysialic acid-neural cell adhesion molecule induces aberrant mossy fiber innervation and ectopic synaptogenesis in the hippocampus. J Neurosci 18:3757-3766.

Smart I (1961) The subependymal layer of the mouse brain and its cell production as shown by radiautography after thymidine-H3 injection. J Comp Neurol 116:325-347.

Sotelo C, Alvarado-Mallart RM, Frain M, Vernet M (1994) Molecular plasticity of adult Bergmann fibers is associated with radial migration of grafted Purkinje cells. J Neurosci 14:124-133.

Tomasiewicz H, Ono K, Yee D, Thompson C, Goridis C, Rutishauser U, Magnuson T (1993) Genetic deletion of a neural cell adhesion molecule variant (N-CAM) produces distinct defects in the central nervous system. Neuron 11:1163-1174.

Wang C, Rougon G, Kiss JZ (1994) Requirement of polysialic acid for the migration of the O-2A glial progenitor cell from neurohypophyseal explants. J Neurosci 14:4446-4457.

Wichterle H, Garcia-Verduge JM, Alvarez-Buylla A (1997) Direct evidence for homotypic, glia-independent neuronal migration. Neuron 18:779-791.

Wood GK, Tomasiewicz H, Rutishauser U, Magnuson T, Quirion R, Rochford J, Srivastava LK (1998) NCAM-180 knockout mice display increased lateral ventricle size and reduced prepulse inhibition of startle. NeuroReport 9:461-466.

Wu W, Wong K, Chen J, Jiang Z, Dupuis S, Wu JY, Rao Y (1999) Directional guidance of neuronal migration in the olfactory system by the protein Slit. Nature 400:331-336. 